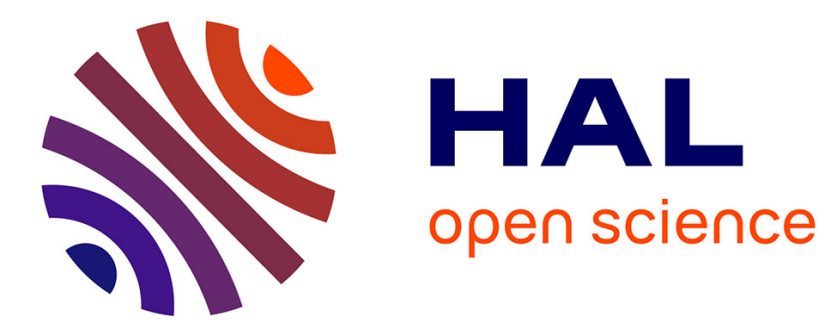

\title{
Cristallogenèse du tellure par la méthode Czochralski
}

\author{
Michel Cerclet
}

\section{To cite this version:}

Michel Cerclet. Cristallogenèse du tellure par la méthode Czochralski. Revue de Physique Appliquée, 1969, 4 (1), pp.63-66. 10.1051/rphysap:019690040106300 . jpa-00242893

\section{HAL Id: jpa-00242893 https://hal.science/jpa-00242893}

Submitted on 1 Jan 1969

HAL is a multi-disciplinary open access archive for the deposit and dissemination of scientific research documents, whether they are published or not. The documents may come from teaching and research institutions in France or abroad, or from public or private research centers.
L'archive ouverte pluridisciplinaire HAL, est destinée au dépôt et à la diffusion de documents scientifiques de niveau recherche, publiés ou non, émanant des établissements d'enseignement et de recherche français ou étrangers, des laboratoires publics ou privés. 


\title{
GRISTALLOGENÈSE DU TELLURE PAR LA MÉTHODE CZOGHRALSKI
}

\author{
Par Miahel CERGLET, \\ Groupement «Physique Électronique et Composants » \\ du Centre National d'Études des Télécommunications, 92-Issy-les-Moulineaux.
}

(Reçu le 7 mai 1968, révisé le 11 décembre 1968.)

\begin{abstract}
Résumé. - On décrit une méthode de préparation de monocristaux de tellure de haute pureté et d'orientation déterminée : fusion en creuset de silice par induction directe en atmosphère d'argon hydrogéné, tirage des cristaux suivant l'axe c. Cette méthode a permis d'obtenir des cristaux pesant jusqu'à $70 \mathrm{~g}$; lorsque la matière première est préalablement purifiée par fusion de zone, la densité de porteurs $p$ est de l'ordre de $10^{14} \mathrm{~cm}^{-3}$ à $77 \mathrm{oK}$. Les cristaux sont finalement recuits à $360{ }^{\circ} \mathrm{C}$.
\end{abstract}

Abstract. - Description of a process of growing tellurium single crystals which are of high quality and have a definite orientation : melting in a silica crucible by direct RF induction in an argon plus hydrogen atmosphere, vertical pulling of the crystals according to the $c$ axis. This method enables one to obtain crystals weighing up to $70 \mathrm{~g}$; when the raw material previously is purified by zone melting, the $p$-type carrier density is about $10^{14} \mathrm{~cm}^{-3}$ at $77 \mathrm{oK}$. The crystals are finally annealed at $360^{\circ} \mathrm{C}$.

I. Introduction. - Le problème posé était celui de la préparation de monocristaux de tellure destinés à des études d'optique non linéaire. Il était nécessaire de disposer de cristaux de diamètre supérieur à $20 \mathrm{~mm}$ (dans ce qui suit, nous appellerons diamètre des cristaux le diamètre du cercle inscrit dans l'hexagone obtenu par une section droite du cristal, celui-ci étant tiré suivant l'axe c). Ainsi que d'autres l'ont déjà fait $[1,2]$, nous avons choisi la méthode Czochralski qui donne des cristaux de bonnes dimensions et d'orientation connue à l'avance.

II. Description de l'appareil. - 1. ENGEINTE DE TIRAGE ET ATMOSPHÈRE. - L'enceinte de tirage est une cloche en acier inoxydable à double paroi refroidie par circulation d'eau. Elle peut être reliée à un ensemble de pompage et à une alimentation de gaz. Elle est munie d'un hublot permettant d'observer le tirage.

Du fait de la forte tension de vapeur du tellure $(0,46$ torr à la température de fusion), et pour retarder son évaporation et la métallisation du hublot d'observation, nous opérons sous atmosphère d'argon contenant $10 \%$ d'hydrogène en surpression de 1 bar environ (pureté du gaz : 99,995\%).

2. Dispositif de chauffage. - Le chauffage est assuré par induction directe dans le bain de tellure. La puissance du générateur H.F. est commandée et stabilisée par un variateur régulateur à amplification magnétique. Cet ensemble permet d'obtenir un chauf- fage suffisamment stable et finement réglable pour permettre des opérations de tirage contrôlées uniquement à la vue, sans mesure ni régulation de température.

Ce moyen de chauffage présente deux avantages principaux :

- l'inertie du chauffage est minimale,

- le creuset n'étant chauffé que par conduction du bain, sa partie supérieure est relativement froide et condense une grande partie du tellure évaporé.

3. Creuset. - Il est en silice synthétique de haute pureté, à fond plat. Il a $60 \mathrm{~mm}$ de diamètre. Un creuset de $40 \mathrm{~mm}$ de diamètre a été utilisé, mais il ne permet pas le tirage de cristaux de diamètre supérieur à $15 \mathrm{~mm}$ environ.

4. Dispositif De tiRAge. - La barre porte-germe en acier inoxydable est refroidie par circulation interne d'eau. Elle est mise en rotation par un moteur muni de réducteurs interchangeables permettant de modifier facilement la vitesse de rotation du germe. Le mouvement de tirage est assuré par une vis mue par un moteur à vitesse variable. La vitesse de tirage peut être réglée d'une manière continue entre 5 et $60 \mathrm{~mm} . \mathrm{h}^{-1}$.

5. Germe. - Il est obtenu par clivage d'un cristal suivant des plans $(10 \overline{1} 0)$ de manière à obtenir un prisme hexagonal de $5 \mathrm{~mm}$ de diamètre et de $50 \mathrm{~mm}$ de longueur environ; on a utilisé également un cristal de 6 à $7 \mathrm{~mm}$ de diamètre. Ce germe est rectifié sur 
quelques millimètres à une extrémité suivant un axe aussi parallèle que possible à l'axe c du cristal. Il semble en effet nécessaire à la réussite du tirage que l'axe de rotation du germe soit confondu avec son axe géométrique et qu'il soit bien perpendiculaire à la surface du bain fondu.

Le germe ainsi préparé s'engage à frottement doux dans un embout en acier inoxydable dans lequel il est

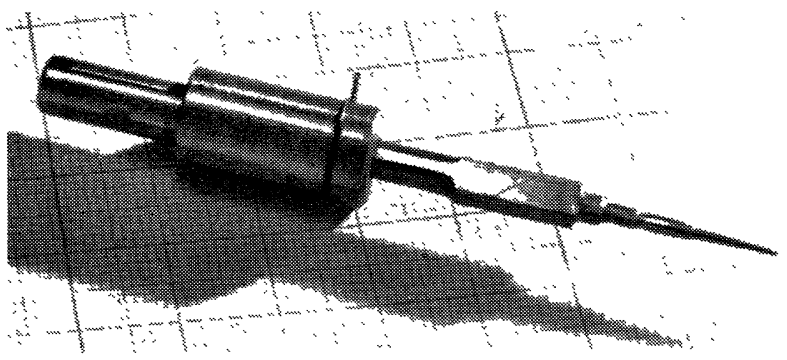

FIG. 1. - Germe fixé dans son embout.

fixé au moyen d'un fil métallique ( fig. 1). Cet embout est fixé par une vis à l'extrémité de la barre portegerme.

III. Conditions opératoires. - 1. VITESSE DE TIRAGE. - Après de nombreux essais à l'intérieur de la gamme citée plus haut, la vitesse de tirage retenue est de 10 à $20 \mathrm{~mm} \cdot \mathrm{h}^{-1}$.

2. Vitesse de rotation. - Les faibles vitesses semblent favorables; la vitesse retenue est de $1 \mathrm{tr} . \mathrm{mn}^{-1}$.

3. Conduite D'une opération. - La température du bain est réglée de manière que le début du cristal soit très fin (diamètre inférieur à $0,5 \mathrm{~mm}$ ) suivant la méthode de Dash, puis la température est abaissée pour obtenir un grossissement assez lent du cristal (angle au sommet de la partie conique de l'ordre de $40^{\circ}$ ), jusqu'à obtention du diamètre désiré. On s'efforce ensuite de maintenir ce diamètre constant. Il est nécessaire, avec notre appareil, d'abaisser progressivement la température jusqu'à la fin de l'opération. La figure 2 montre un cristal ainsi obtenu qui n'a pas encore été séparé de son germe.

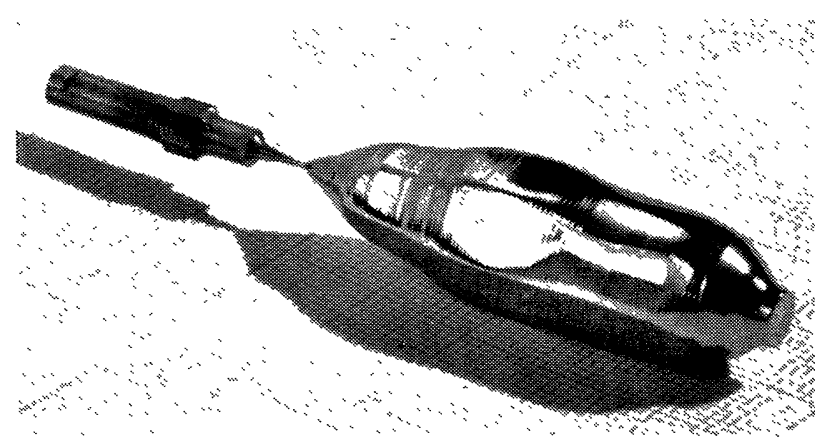

FIG. 2. - Cristal de tellure non séparé de son germe
IV. Résultats obtenus. - 1. Dimensions DES GRISTAUx. - On a tiré des cristaux de diamètre variant entre 4 et $23 \mathrm{~mm}$ (dans la partie cylindrique) et pesant jusqu'à $70 \mathrm{~g}$ ( $\mathrm{fg}$. 2 et 3 ). Ces dernières valeurs

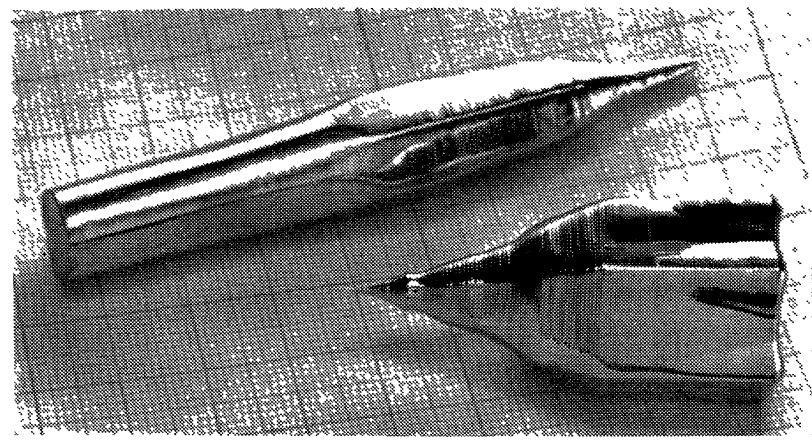

FIG. 3. - Cristaux de tellure.

semblent être les maximums qu'il soit possible d'obtenir avec le creuset décrit plus haut. Le diamètre minimal du cristal de $70 \mathrm{~g}$ était de $0,2 \mathrm{~mm}$.

2. Qualité cristalline. - On peut penser que les cristaux bruts de tirage présentent des contraintes internes élevées qui perturbent le réseau cristallin, ce qui est confirmé, dans l'examen aux rayons $\mathrm{X}$, par la déformation d'une réflexion de Bragg (diagramme de Lambot) (fig. 4 a). Un recuit semble donc nécessaire.
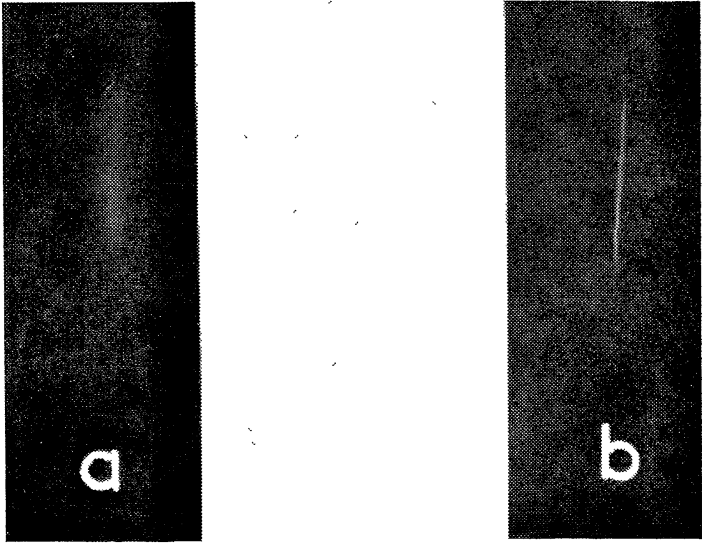

FIG. 4. - Diagramme de Lambot obtenu sur un cristal de tellure :

a) Brut de tirage.

b) Recuit.

Les publications sur la question indiquent des conditions de recuit très différentes suivant les auteurs : entre autres de $70 \mathrm{~h}$ à $320^{\circ} \mathrm{C}$ [4] jusqu'à $168 \mathrm{~h}$ à $380^{\circ} \mathrm{C}$ [6]. En conséquence, nous avons fait une série de recuits de durées croissantes à la température de $360^{\circ} \mathrm{C}$ en contrôlant l'effet obtenu par l'aspect du diagramme de Lambot. La figure $4 \mathrm{~b}$ montre l'aspect 
obtenu après 27 h. En attendant une étude plus poussée, nous avons retenu le programme de recuit suivant : montée à $360^{\circ} \mathrm{C}$ en $10 \mathrm{~h}$, palier pendant $20 \mathrm{~h}$, descente jusqu'à la température ambiante en $18 \mathrm{~h}$. Le recuit est fait sous vide $\left(10^{-3}\right.$ torr $)$ en ampoule de Pyrex.

Les dislocations ont été révélées par voie chimique sur les plans $(10 \overline{1} 0)$; le réactif, du type utilisé par Lovell, Wernick et Benson [7], a la composition suivante :

$\begin{array}{lll}-\mathrm{HF} & : 3 \text { volumes } \\ \text { - } \mathrm{HNO}_{3} & : 50- \\ -\mathrm{HCH}_{3} \mathrm{CO}_{2} & : 6 & -\end{array}$

En général, la densité de dislocations est forte, de l'ordre de $10^{5}$ à $10^{6} \mathrm{~cm}^{-2}$; certains cristaux cependant ne dépassent pas $3 \times 10^{4} \mathrm{~cm}^{-2}$.

Une étude plus poussée des dislocations est en cours, en particulier sur le rôle de divers facteurs : recuit, traitements mécaniques, clivage à différentes températures.

3. Propriétés élegtriques et magnétiques. - On a mesuré la résistivité et la constante de Hall en fonction de l'inverse de la température sur un échantillon non recuit, de section trapézoïdale, obtenu par clivage parallèlement à l'axe $\mathrm{c}$, à la température de l'azote liquide et dont les dimensions sont les suivantes :

- longueur (parallèle à l'axe c) : $11 \mathrm{~mm}$,

- épaisseur : 1,3 $\mathrm{mm}$,

- largeur : grande base $3 \mathrm{~mm}$, petite base $1,5 \mathrm{~mm}$.

Afin d'éliminer les perturbations de surface dues au clivage, on enlève sur l'échantillon une épaisseur de $20 \mu$ environ au moyen du réactif chromique indiqué par Blakemore, Schultz et Nomura [3].

Enfin, six sondes en fil de cuivre de $0,1 \mathrm{~mm}$ de diamètre sont soudées à l'échantillon au moyen de l'alliage Sn $50 \%$-In $50 \%$ : une à chaque extrémité et quatre latéralement au tiers de la longueur.

Les résultats de ces mesures sont portés sur les courbes de la figure 5. On a obtenu :

- température d'inversion de type : 178 oK;

$$
\begin{aligned}
& \text { — à } 300 \mathrm{oK}: R=510 \mathrm{~cm}^{3} \cdot \mathrm{C}^{-1} \\
& \rho=0,395 \Omega . \mathrm{cm} \text {; } \\
& \text { - à } 77 \mathrm{oK}: R=28200 \mathrm{~cm}^{3} \cdot \mathrm{C}^{-1} \\
& \rho=16,3 \Omega . \mathrm{cm}
\end{aligned}
$$

on en déduit une densité de porteurs $p$ :

$$
N_{\mathrm{a}}=2,2 \times 10^{14} \mathrm{~cm}^{-3}
$$

et la mobilité $\mu=1730 \mathrm{~cm}^{2} \mathrm{~V}^{-1} \mathrm{~s}^{-1}$.

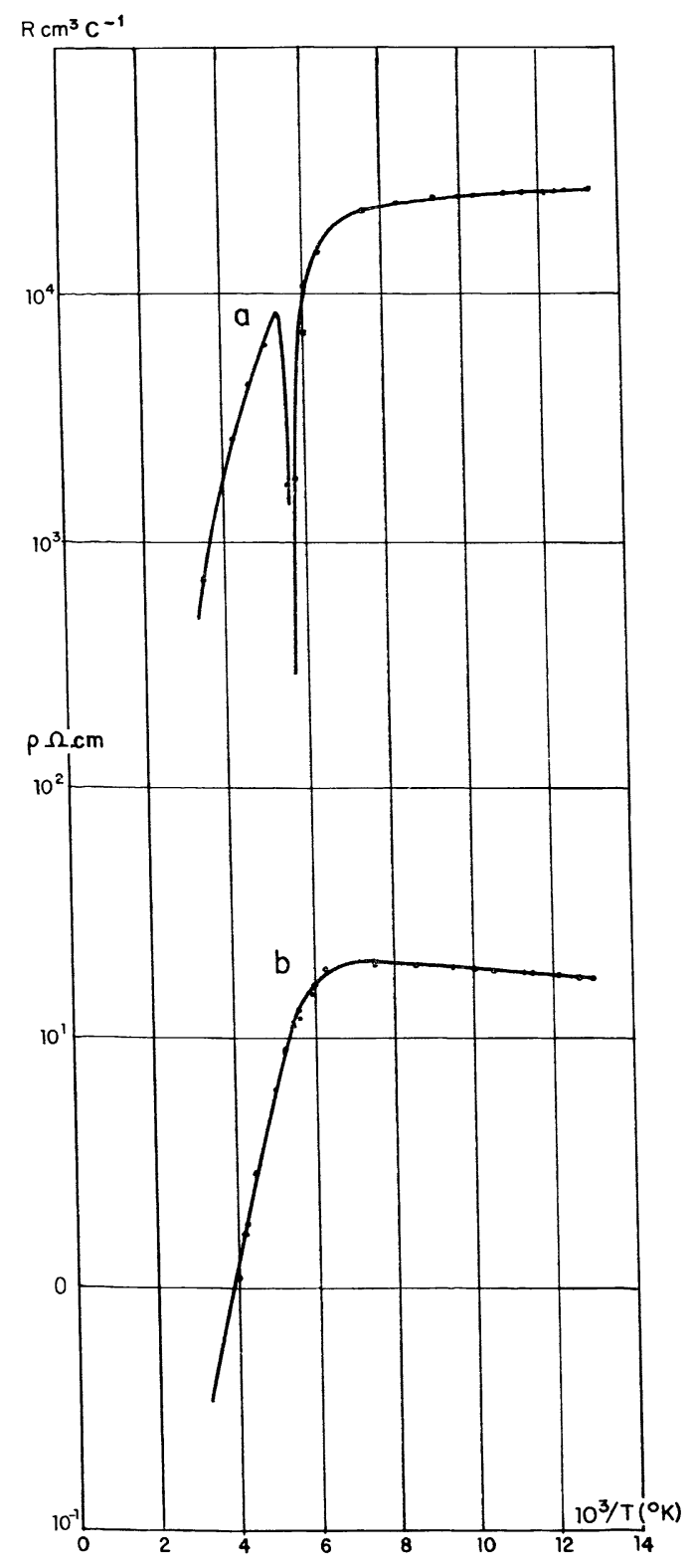

FIG. 5. - a) Constante de Hall du tellure en fonction de l'inverse de la température ; b) Résistivité du tellure en fonction de l'inverse de la température parallèlement à l'axe c.

V. Conclusion. - Nous avons rencontré quelques difficultés au début de cette étude car nous ne disposions que de germes petits et de qualité médiocre. Actuellement, cette méthode permet d'obtenir d'une manière parfaitement reproductible des cristaux de plus de $80 \mathrm{~mm}$ de long ou de plus de $20 \mathrm{~mm}$ de diamètre, pesant jusqu'à $70 \mathrm{~g}$.

Après purification du tellure commercial par fusion de zone, nous avons tiré des cristaux dont la 
densité de porteur $p$ est de $2,2 \times 10^{14} \mathrm{~cm}^{-3}$ à $77^{\circ} \mathrm{K}$. Le recuit de ces cristaux améliore grandement leur qualité cristalline.

Ce travail a été effectué sous la direction de M. Petit
Le Du. Noùs remercions vivement Mme Soula et M. Pécile pour les mesures de densité de dislocations et de propriétés électriques qu'ils ont bien voulu faire sur nos cristaux.

\section{BIBLIOGRAPHIE}

[1] WEIDEL (J.), Z. Naturforsch., 1954, 9 a, 697.

[2] Davies (T. J.), J. Appl. Phys., 1957, 28, 1217.

[3] Blakemore (J. S.), SchulTz (J. W.) et Nomura (K. C.), J. Appl. Phys., 1960, 31, 2226.

[4] PARFEn'Ev (R. V.), FARbShTEIN (I. I.) et ShaI.Y'T (S. S.), Sov. Phys. Solid State, 1961, 2, 2599.
[5] Kozhitov (L. V.), Vanjukov (A. V.) et Shapkin (P. V.), Tsvet Métal, 1967, 40, 76.

[6] Korovin et Firsov, Sov. Phys. Techn. Phys., 1958, 3, 2219 .

[7] Loveli (L. C.), Wernick (J. H.) et Benson (K. E.), Acta Met., 1958, 6, 716. 